Research Paper

\title{
Chemoradiotherapy for Synchronous Multiple Primary Cancers with Esophageal Squamous Cell Carcinoma: a Case-control Study
}

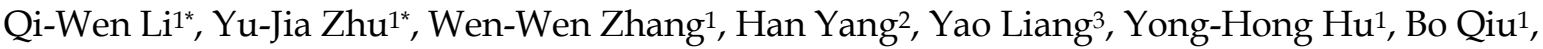 \\ Meng-Zhong Liu ${ }^{1}$, Hui Liu ${ }^{1 凶}$
}

1. Departments of Radiation Oncology, State Key Laboratory of Oncology in South China, Collaborative Innovation Center for Cancer Medicine, Sun Yat-sen University Cancer Center, Guangzhou, Guangdong, China;

2. Departments of Thoracic Oncology, State Key Laboratory of Oncology in South China, Collaborative Innovation Center for Cancer Medicine, Sun Yat-sen University Cancer Center, Guangzhou, Guangdong, China;

3. Departments of Gastric and Pancreatic Surgery, State Key Laboratory of Oncology in South China, Collaborative Innovation Center for Cancer Medicine, Sun Yat-sen University Cancer Center, Guangzhou, Guangdong, China.

${ }^{*}$ Qi-Wen Li and Yu-Jia Zhu contributed equally to this work.

$\triangle$ Corresponding author: Hui Liu, Department of Radiation Oncology, Sun Yat-sen University Cancer Center, No.651 Dongfeng Road East, Guangzhou, 510060, China. E-mail: liuhui@sysucc.org.cn. Tel: +862087343031. Fax number: +862087343492.

(C) Ivyspring International Publisher. This is an open access article distributed under the terms of the Creative Commons Attribution (CC BY-NC) license (https://creativecommons.org/licenses/by-nc/4.0/). See http://ivyspring.com/terms for full terms and conditions.

Received: 2016.08.31; Accepted: 2016.10.29; Published: 2017.02.11

\begin{abstract}
Objective: To evaluate the efficacy and toxicity of concurrent chemoradiotherapy (CRT) in multiple primary cancers (MPC) of the upper digestive tract in esophageal squamous cell carcinoma (ESCC).

Methods: In a screening of 1193 consecutive patients diagnosed with ESCC and received radiotherapy, 53 patients presenting synchronous MPC in the upper digestive tract were retrospectively investigated. 53 consecutive patients with esophageal non-multiple primary cancer (NPC), matched by stage, age and sex, served as control. All of the patients received concurrent CRT. The median radiation dose was 60 Gy. Chemotherapy regimens were based on platinum and/or 5-fluorouracil. Clinical outcomes and treatment toxicities were compared.

Results: Clinic-pathologic characteristics were well balanced between groups. MPC mostly located in esophagus $(43,81.8 \%)$, followed by hypopharynx $(8,15.1 \%)$ and stomach $(2,3.8 \%)$. In MPC and NPC patients, $94.3 \%$ and $96.2 \%$ completed the intended treatment. The immediate response rate was $73.6 \%$ vs $75.5 \%$, with complete response rate of $11.3 \%$ vs $24.5 \%$ and partial response rate of $62.3 \%$ vs $51.0 \%$. Two-year overall survival (OS), progression-free survival (PFS), locoregional progression-free survival (LRPFS) and distant progression-free survival (DPFS) were $52.2 \%$ vs $68.9 \%(p=0.026), 32.9 \%$ vs $54.0 \%$ $(p=0.032), 60.8 \%$ vs $87.8 \%(p=0.002)$ and $64.0 \%$ vs $70.8 \%(p=0.22)$, respectively. Acute grade $3-4$ toxicities were observed in $64.2 \%$ vs $54.7 \%$, significantly higher in radiation esophagitis $(49.1 \%$ vs $28.3 \%$, $p<0.001)$, and mucositis ( $11.3 \%$ vs $00 p=0.027)$.

Conclusions: Compared with matched NPC, ESCC accompanied with synchronous MPC was related to significantly impaired survival, elevated risk of locoregional disease progression and higher incidence of severe esophagitis and mucositis, following concurrent chemoradiotherapy. Future study on reasons for decreased efficacy of chemoradiotherapy will help to optimize treatment. Advanced radiation techniques may play a role in protecting normal tissues and reduce acute toxicities.
\end{abstract}

Key words: esophageal cancer, second primary malignancy, chemoradiotherapy, survival, toxicity

\section{Introduction}

Multiple primary cancers (MPC) often arise simultaneously or metachronously with esophageal squamous cell carcinoma (ESCC). Most MPC occur in the aerodigestive tract, including the head-and-neck region, lungs and esophagus, with an incidence from $7.7 \%$ to $20.7 \%$ [1-4]. According to Warren and Gates, 
when MPC develop within 6 months after the diagnosis of the index cancer, they are considered to be synchronous [5], and these accounts for about half of all MPC with esophageal cancers [2-4, 6].

Demonstrated by previous studies, MPC and esophageal non-multiple primary cancer (NPC) were of distinct characteristics, as a reflection of different gene background and environmental conditions [7]. The complexity of the tumor type, as well as different location and stage of MPC, has constrained a consensus on the optimal treatment. Regretfully, attempts on extensive surgery have shown limited R0 resection rates and variating clinical outcomes [8-10]. Though several reports have focused on the efficacy and toxicity of concurrent chemoradiotherapy (CRT) [11-13], a direct comparison between MPC and NPC patients, with balanced baseline characteristics and relatively homogenous treatment modalities, had not been available. Thus, we designed the retrospective study to determine the efficacy and toxicity of concurrent CRT in patients with synchronous MPC and ESCC, with comparison by NPC counterparts.

\section{Materials and Methods}

The study was reviewed and approved by the institutional review board of Sun Yat-sen University Cancer Center and conducted according to the principles expressed in the Declaration of Helsinki. Because the study was a retrospective and anonymous analysis of routine data, we requested and were granted a waiver of individual informed consent.

\section{Patient characteristics}

From Jan 2011 to May 2015, a total of 1193 consecutive patients with a pathological diagnosis of ESCC and who received radiotherapy at Sun Yat-sen University Cancer Center were retrospectively screened. Among this cohort, 53 patients were identified as having at least one synchronous MPC in the upper digestive tract and underwent concurrent CRT. The standards proposed by Warren and Gates were satisfied: (1) all tumors were histologically confirmed; (2) all lesions were anatomically discontinuous and surrounded by normal tissue; and (3) distant metastases were excluded [5]. Tumors were diagnosed synchronously within an interval not exceeding 6 months. Clinical staging was based on the American Joint Committee on Cancer 2002 Criteria and consisted of comprehensive evaluations that included barium swallow, endoscopic ultrasonography (EUS), computed tomography (CT) of the neck, chest and abdomen, as well as MRI of the head and neck area or whole-body PET/CT, if necessary. Metastatic lymph nodes were considered as regional nodes of the primary ESCC if located in its drainage area; otherwise, they were evaluated as positive nodes of MPC, or distant metastasis if they were outside the region of both neoplasms.

Another 53 patients with single pathological diagnosed ESCC, who were treated with concurrent chemoradiotherapy during the same period, were consecutively included. Essential parameters, including stage, age and sex were manually matched. These patients were considered as the control group.

\section{Treatments}

All patients received external beam radiation and concurrent chemotherapy, with or without systemic neoadjuvant chemotherapy. Threedimensional conformal radiotherapy (3D-CRT), intensity-modulated radiotherapy (IMRT) or volumetric modulated arc therapy (VMAT) were adopted using a 6-8 MV photon beam and conventional fraction (1.8-2.4 Gy). At least 50 Gy was delivered to primary esophageal cancer. During radiotherapy, chemotherapy regimens were consisted either of a single agent of platinum or 5-fluorouracil (5-FU) or of a double-drug combination of taxane+platinum (TP) or platinum +5 -fluorouracil (PF), given every week (platinum $25 \mathrm{mg} / \mathrm{m}^{2}$ or $5-\mathrm{FU}$ $1.33 \mathrm{~g} / \mathrm{m}^{2}$ civ 72 hours or taxane $25 \mathrm{mg} / \mathrm{m}^{2}+$ platinum $25 \mathrm{mg} / \mathrm{m}^{2}$ or platinum $25 \mathrm{mg} / \mathrm{m}^{2}+5$-fluorouracil 1.33 $\mathrm{g} / \mathrm{m}^{2}$ civ 72 hours, $\mathrm{d} 1, \mathrm{~d} 8, \mathrm{~d} 15, \mathrm{~d} 22$ ) or every three weeks (platinum $75 \mathrm{mg} / \mathrm{m}^{2}$ or 5 -FU $4.0 \mathrm{~g} / \mathrm{m}^{2}$ civ 120 hours or taxane $75 \mathrm{mg} / \mathrm{m}^{2}+$ platinum $75 \mathrm{mg} / \mathrm{m}^{2}$ or platinum $75 \mathrm{mg} / \mathrm{m}^{2}+5$-fluorouracil $4.0 \mathrm{~g} / \mathrm{m}^{2}$ civ 120 hours, d1, d22).

\section{Response evaluation and follow-up}

Comprehensive re-evaluation work-ups conducted 2 months after the completion of CRT were studied by an experienced radiation oncologist. Responses were assessed according to Response Evaluation Criteria in Solid Tumors version 1.1. Acute and late toxicities were collected retrospectively and presented on the basis of Radiation Therapy Oncology Group grading. Follow-up evaluation was performed every 3 months during the first 2 years after treatment, every 6 months in the third year and once a year thereafter, including the examinations mentioned above.

\section{Study end points}

The primary endpoint was overall survival (OS) and treatment toxicity. The secondary endpoints were progression-free survival (PFS), locoregional progression-free survival (LRPFS) and distant progression-free survival (DPFS). OS was defined as the time from initial diagnosis (for both primary and secondary cancers) to death from any cause. PFS was 
defined as the time from diagnosis to disease progression or death. Locoregional progression was defined as progression occurring in the original location or regional lymph nodes, while other progression was considered as distant progression.

\section{Statistical methods}

Survival was assessed by the Kaplan-Meier method and compared using a log-rank test. Comparisons of categorical variables between groups were performed by Fisher's exact test. P-value $<0.05$ (two-sided) was considered statistically significant. All tests were conducted using SPSS 21.0.

\section{Results}

The incidence of MPC in patients diagnosed with ESCC and who received CRT in our hospital was 4.4\% (53/1193). All malignant lesions, including NPC and MPCs, were pathological confirmed as squamous cell cancer. Out of 53 patients, $45(84.9 \%)$ in MPC group were diagnosed with double cancers simultaneously. Others received their diagnoses between 3-21 days; with no therapy prior to complete assessments of multiple primary cancers. Clinico-pathologic characteristics are presented in Table 1. Of all $53 \mathrm{MPC}$ cases, $90.6 \%$ were male. Tobacco and alcohol consumption were documented $66.0 \%$ and $49.1 \%$ among this population. A family history of malignancy was identified in 26.4\%. 10 (18.9\%) patients were identified as underweight according to body mass index (BMI). MPCs were mainly found in esophagus $(43,81.8 \%)$, with less in the hypopharynx $(8,15.1 \%)$ and stomach $(2,3.8 \%)$. The median radiation dose delivered for primary esophageal cancer and MPC was 60 Gy (range: 50-67 Gy for primary esophageal cancer and 46-67 Gy for MPC). Concomitantly, 47 (88.7\%) patients received TP or PF, and $6(11.3 \%)$ received a single-agent regimen (platinum or 5-FU). Neoadjuvant chemotherapy was adopted in $20(37.7 \%)$ patients, with two as the median number of cycles. Baseline clinic-pathologic characteristics were statistically balanced between MPC and NPC groups.

\section{Toxicities}

In MPC group, grade 3-4 acute toxicities were reported in $34(64.2 \%)$ patients and predominately consist of esophagitis (26 patients, $49.1 \%$ ) and myelosuppression (18 patients, 34.0\%), followed by 6 $(11.3 \%)$ cases of severe mucositis, $4(6.1 \%)$ cases of severe pneumonia, and other grade 3-4 adverse effects (Table 2). Fifty $(94.3 \%)$ patients completed the intended radiotherapy, while treatment was discontinued in 2 patients due to esophagitis, lowe to aspiration pneumonitis. For NPC patients, grade 3-4 acute toxicities were observed in $29(54.7 \%)$ patients $(p=0.43)$. The incidence of severe esophagitis (15 patients, $28.3 \%, p<0.001)$ and mucositis (0 patients, $p=0.027$ ) was significantly lower, while similar for myelosuppression (21 patients, 39.6\%, $p=0.69$ ), and pneumonia ( 1 patients, $1.9 \%, p=0.36$ ). The treatment completion rate was $96.2 \%$, with 2 patients stopped radiation prematurely because of grade 3 pneumonia and grade 4 laryngitis.

No severe late toxicities were recorded.

Table 1. Clinico-pathologic characteristics

\begin{tabular}{|c|c|c|c|c|c|}
\hline \multirow[t]{2}{*}{ Characteristics } & \multicolumn{2}{|c|}{ MPC } & \multicolumn{2}{|c|}{ NPC } & \multirow[b]{2}{*}{$p$} \\
\hline & No. & $\%$ & No. & $\%$ & \\
\hline \multicolumn{6}{|l|}{ Age } \\
\hline$\geq 60$ & 24 & 45.3 & 27 & 50.9 & \multirow[t]{2}{*}{0.56} \\
\hline$<60$ & 29 & 53.7 & 26 & 49.1 & \\
\hline \multicolumn{6}{|l|}{ Sex } \\
\hline Male & 48 & 90.6 & 42 & 79.2 & \multirow[t]{2}{*}{0.17} \\
\hline Female & 5 & 9.4 & 11 & 20.8 & \\
\hline \multicolumn{6}{|c|}{ Location of primary esophageal cancer } \\
\hline Cervix & 16 & 30.2 & 7 & 13.2 & \multirow[t]{4}{*}{0.14} \\
\hline Upper $1 / 3$ esophagus & 16 & 30.2 & 15 & 28.3 & \\
\hline Middle $1 / 3$ esophagus & 19 & 35.8 & 27 & 50.9 & \\
\hline Lower $1 / 3$ esophagus & 2 & 3.8 & 4 & 7.5 & \\
\hline \multicolumn{6}{|c|}{ Clinical stage of primary esophageal cancer } \\
\hline I-II & 7 & 13.2 & 7 & 13.2 & \multirow[t]{2}{*}{1.0} \\
\hline III-IV & 46 & 86.8 & 46 & 86.8 & \\
\hline \multicolumn{6}{|l|}{ KPS } \\
\hline$\geq 90$ & 44 & 83.0 & 43 & 81.1 & \multirow[t]{2}{*}{1.0} \\
\hline$<90$ & 9 & 17.0 & 10 & 18.9 & \\
\hline \multicolumn{6}{|l|}{ Smoking } \\
\hline Yes & 35 & 66.0 & 37 & 69.8 & \multirow[t]{2}{*}{0.84} \\
\hline No & 18 & 34.0 & 16 & 30.2 & \\
\hline \multicolumn{6}{|l|}{ Drinking } \\
\hline Yes & 26 & 49.1 & 23 & 43.4 & \multirow[t]{2}{*}{0.70} \\
\hline No & 27 & 50.9 & 30 & 56.6 & \\
\hline \multicolumn{6}{|l|}{ Family history } \\
\hline Yes & 14 & 26.4 & 9 & 17.0 & \multirow[t]{2}{*}{0.35} \\
\hline No & 39 & 73.6 & 44 & 83.0 & \\
\hline BMI & & & & & \\
\hline$<18.5$ & 10 & 18.9 & 8 & 15.1 & 0.80 \\
\hline$\geq 18.5$ & 43 & 81.1 & 45 & 84.9 & \\
\hline Concurrent chemothera & & & & & \\
\hline $\mathrm{TP} / \mathrm{PF}$ & 47 & 88.7 & 49 & 92.5 & 0.74 \\
\hline 5-FU/platinum & 6 & 11.3 & 4 & 7.5 & \\
\hline Induction chemotherap & & & & & \\
\hline Yes & 20 & 37.7 & 12 & 22.6 & 0.14 \\
\hline No & 33 & 62.3 & 41 & 77.4 & \\
\hline RT technique & & & & & \\
\hline 3D-CRT & 11 & 20.8 & 18 & 34.0 & 0.19 \\
\hline IMRT/VMAT & 42 & 79.2 & 35 & 66.0 & \\
\hline RT dose for primary es & & & & & \\
\hline$\geq 60 \mathrm{~Gy}$ & 35 & 66.0 & 40 & 75.5 & 0.39 \\
\hline$<60$ Gy & 18 & 34.0 & 13 & 24.5 & \\
\hline Location of second prin & & & & & \\
\hline Esophagus & 43 & 81.1 & & & \\
\hline Hypopharynx & 8 & 15.1 & & & \\
\hline Stomach & 2 & 3.8 & & & \\
\hline Clinical stage of second & & & & & \\
\hline I-II & 34 & 64.2 & & & \\
\hline III-IV & 19 & 35.8 & & & \\
\hline RT dose for second prir & & & & & \\
\hline$\geq 60$ Gy & 33 & 62.3 & & & \\
\hline$<60 \mathrm{~Gy}$ & 20 & 37.7 & & & \\
\hline
\end{tabular}


Table 2 Acute grade 3-4 toxicities.

\begin{tabular}{llllll}
\hline Toxicity & MPC & \multicolumn{5}{l}{ NPC } \\
\hline & Grade 3 & Grade 4 & Grade 3 & Grade 4 & $p$ (Grade3+4) \\
\hline Esophagus & 31 & 3 & 12 & 3 & $<0.001$ \\
Neutropenia & 6 & 8 & 7 & 6 & 1.0 \\
Thrombocytopenia & 7 & 0 & 6 & 2 & 1.0 \\
Anemia & 4 & 0 & 0 & 1 & 0.36 \\
Mucous membrane & 5 & 1 & 0 & 0 & 0.027 \\
Lung & 4 & 0 & 1 & 0 & 0.36 \\
Larynx & 1 & 0 & 1 & 1 & 1.0 \\
Upper gastrointestinal & 1 & 0 & 0 & 0 & 1.0 \\
tract & & & & & \\
Lower gastrointestinal & 1 & 0 & 0 & 0 & 1.0 \\
tract & & & & & \\
Eye & 1 & 0 & 0 & 0 & 1.0 \\
\hline
\end{tabular}

MPC: multiple primary cancers; NPC: non-multiple primary cancer.

\section{Clinical outcomes}

The response rate at 2 months after completion of CRT in MPC and NPC patients were $73.6 \%$ (39) vs $75.5 \%$ (40), with $11.3 \%$ (6) vs $24.5 \% \quad$ (13) complete responses and $62.3 \%$ (33) vs $51.0 \%$ (27) partial responses. Stable disease and progressive disease was found in $18.9 \%$ (10) vs $20.8 \%$ (11) and $7.5 \%$ (4) vs $3.8 \%$ (2), respectively. The response between two groups was not statistical significant $(p=0.28)$.

In MPC patients, at the median follow-up of 16 (range: 2-115) months, 36 (67.9\%) patients experienced disease progression, $12(22.6 \%)$ locoregional, 15 (28.3\%) distant, 4 (7.5\%) both, and 5 (9.4\%) unknown.
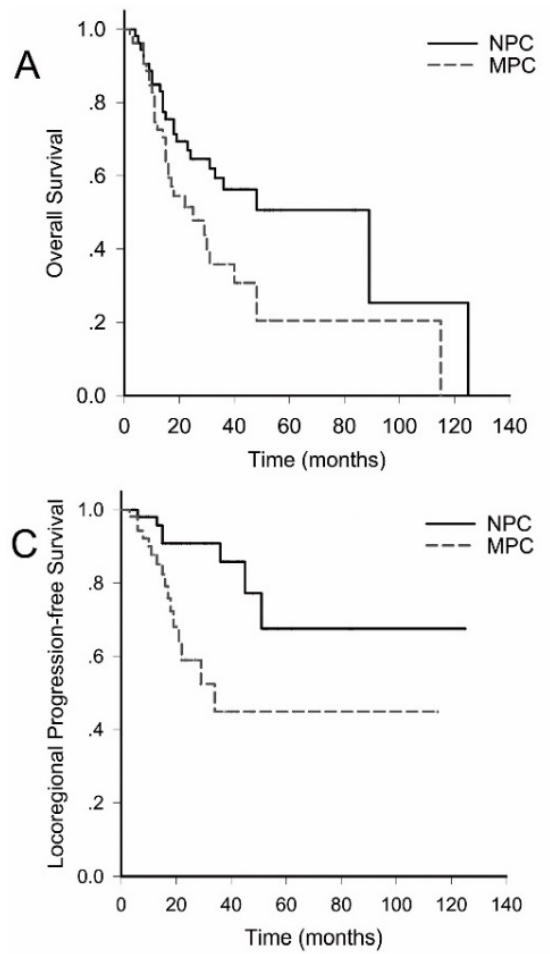

One patient with regional recurrence underwent neck dissection followed by irradiation. One patient received stereotactic body radiotherapy for solitary lung metastasis. Eight patients were prescribed chemotherapy, and the others were given best supportive treatment. At the last evaluation, there were $30(56.6 \%)$ deaths, all but one due to disease progression.

For NPC patients, at the median follow-up of 24 (range: 4-125) months, 29 (54.7\%) experienced disease progression, 6 (11.3\%) locoregional, 15 (28.3\%) distant, 1 (1.9\%) both, and 7 (13.2\%) unknown. Nine patients were given chemotherapy, and the others received best supportive treatment. At the last evaluation, 24 $(45.3 \%)$ deaths were recorded, all owe to disease progression.

As calculated, the two-year overall survival (OS), progression-free survival (PFS), locoregional progression-free survival (LRPFS) and distant progression-free survival (DPFS) were $52.2 \%$ vs $68.9 \%$ $(p=0.026), 32.9 \%$ vs $54.0 \%$ ( $p=0.032), 60.8 \%$ vs $87.8 \%$ $(p=0.002), 64.0 \%$ vs $70.8 \%(p=0.22)$, respectively (Fig. 1).

Based on univariate analyses (Supplementary Table 1), male patients tended to have unfavorable PFS than female patients (2-year PFS: $36.4 \%$ vs $68.8 \%$, $p=0.026$ ).
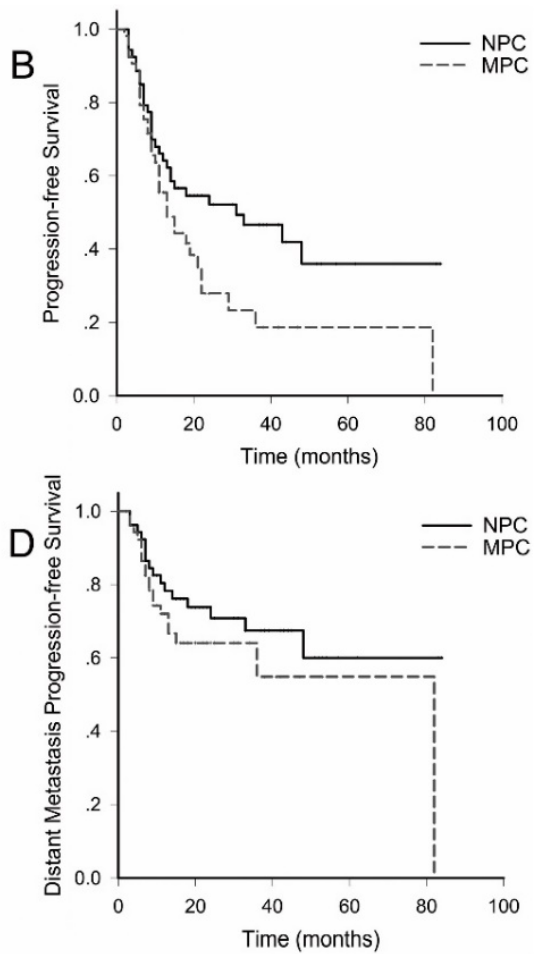

Figure 1. Clinical outcomes. Overall survival; B. Progression-free survival; C. Locoregional progression-free survival; D. Distant progression-free survival. MPC: multiple primary cancers; NPC: non- multiple primary cancer 


\section{Discussions}

Consistent with the range, $4.1-10.5 \%$, as reported previously [2-4, 6], the incidence of synchronous MPC in the upper digestive tract in patients initially diagnosed with ESCC and who underwent radiation in our hospital was $4.4 \%(66 / 1485)$. According to current knowledge, tobacco and alcohol abuse were the most cited features for SPM following head-and-neck squamous cell cancer [7]. There was also evident that lower BMI and HPV infection [14] were suspected risk factors. In our study, because of the way of matching, the distribution of smoking, drinking and BMI was not significantly different between groups, but noticed as a tendency. SPMs were located mainly within esophagus, followed by hypopharynx, which was also in good accordance with previous reports [15].

In addition to environmental or life-style associated factors, family history of malignancy was traced in $26.4 \%$ in the current study, suggesting a potential role of gene mutations and polymorphisms in the development of MPC [16-18]. Various types of gene alteration, i.e. loss of heterozygosity of p53, p16 and IRF, and genetic polymorphisms of aldehyde dehydrogenase type 2 (ALDH2), were already discussed, though none of specific genetic pathways were accredited to be responsible for such "field cancerization phenomenon" [19-20]. Recently, the relation between low $\zeta$-chain expression in T-cells and MPC addressed the role of suppressed immune function in initialing multiple cancers, also featuring the effect of internal environment on MPC generation [21].

Previous studies comparing the prognosis of MPC and NPC have yielded conflicting results [1,22]. Most of researches included heterogeneous populations, with either unbalanced clinic-pathologic characteristics, or various therapeutic strategies. Our study, matching patients by stage, age and sex, has made direct comparison of MPC and NPC of esophageal cancer. As a result of concurrent CRT, the early response rate was similar, but significantly impaired OS, PFS as well as LRPFS (2-year OS: 52.2\% vs $68.9 \%$, $p=0.026$; 2 -year PFS: $32.9 \%$ vs $54.0 \%, p=0.032$; 2-year LRPFS: $60.8 \%$ vs $87.8 \%, p=0.002$ ) in MPC group was identified. In other words, though comparable early response rate had implied similar radiosensibility, MPC was more likely to progress, reappear or generate within 2 years. In a parallel study, 34 patients with localized synchronous head-and-neck squamous cell carcinoma and esophageal cancer were treated with concurrent CRT. Median radiation doses for head-and-neck cancer and esophageal cancer were 70 (60-70.5) Gy and 60 (45-70)
Gy. As a consequence, 2-year overall survival and disease-free survival were $44 \%$ and $33 \%$ [11], comparable to those of the current study. Another study from Nguyen et al. reported that although $66 \%$ of cases achieved clinical complete remission at the end of neoadjuvant chemotherapy followed by radiotherapy, more than half of patients had local relapse and died from their tumor. The median survival was 12 months, with 2 -year OS of only $5 \%$ [12]. In contrast, Welz et al. revealed a favorable median OS reaching to 37 months, but the stage of esophageal cancer tended to be earlier than that of our study [13]. What are the hypotheses for notably increased locoregional progression in MPC patients? First, specific genetic background may contribute to aggressive phenotype. Second, insufficient treatment intensity was under suspicion. Further studies are expected to focus on analyzing the detailed pattern of locoregional relapse to modify the current target volume and dose prescription. Third, it is natural that the presence of two or more potentially lethal tumors leads to an increased chance of relapse or dying from diseases. Fourth, persistent addiction to alcohol and tobacco may occur more frequently in MPC patients, facilitating the generation of a de novo tumor.

As a substitute of local therapy, there were uncertainties regarding the efficacy and adverse effects for surgery. Some authors figured out, even if the rate of radical resection of esophageal lesion was comparable, the presence of additional head and neck tumors was associated with a worse long-term prognosis [8]. According to Lee et al., when half of the resections were radical, 5-year survival was only $9.2 \%$ in patients receiving surgery for esophageal and head-and-neck cancer [9]. However, Otowa, Y. et al. addressed no differences in OS in patients with or without MPC receiving surgery in a non-randomized controlled study, but the extraordinarily high 5-year survival indicated possible strict selection of participants [10]. On the other hand, patients must be carefully evaluated before the decision of operation, because extremely invasive and complicated surgical technique, i.e. pharyngogastric or colonic anastomosis, microvascular anastomosis, myocutaneous flaps, or free jejunal grafts was sometimes required for $\mathrm{R} 0$ resection [22], which may induce increased risk of morbidity [23]. Thus, the decision of surgical resection should be made cautiously. In difficult cases, chemoradiotherapy served as an optimal alternative.

The prognostic factor of SPM and ESCC remained unresolved. In the current study, male patients tended to have worse PFS. Some authors believed that the primary esophageal cancer was the predominant determinant of survival since it was 
often more advanced at diagnosis, compared with synchronous cancer arising in other sites of the upper digestive tract [11]. These may leave some hints on the treatment optimization of MPC, supporting local therapy on esophageal as a priority. Recently, in synchronous squamous cell carcinoma in the upper areo-digestive tract, concomitantly elevated matrix metalloproteinases 3 and 9 levels turned out to be useful predictive biomarkers, even overcame TNM staging [24]. These findings also triggered interest on prognostic stratification on the basis of molecular variation and facilitate individualized treatment. However, the hypotheses should be considered cautiously and be tested by further large scale, strictly controlled studies.

Acute toxicities, especially those non-hematologic, appeared to be a major concern in our study. The incidence of severe acute grade 3-4 esophagitis and mucositis was significantly higher in MPC, as a result of extensive radiated mucosa, and usually followed by malnutrition and loss of weight. The frequent application (86.3\%) of two-drug chemotherapy as the concurrent regimen may emerge as another reason. When single-drug regimens are administered concomitantly, severe toxicities have been reported in only $21 \%$ of patients and primarily involve myelosuppression [11]. Since low BMI has been identified as a significant inverse prognostic factor [25], how to reduce treatment toxicity became a vital question. High expectations were placed on more advanced radiation techniques, i.e., tomotherapy, proton therapy or heavy ion radiotherapy, adjusted concomitant chemotherapy, as well as positive nutritional support.

In the current study, we used matching to compare the prognosis of MPC and NPC following concurrent chemoradiotherapy. Since sex was one of the matched factors while drinking and smoking are sex-relevant, their distribution between MPC and NPC patients were artificially affected. Another deficiency to aware was the undetailed record of location of recurrence, which will be concerned in future studies, to analyze the association of target volume, dose prescription and relapse.

In conclusion, concurrent CRT for synchronous MPC in the upper digestive tract and esophageal cancer, based on the precision radiotherapy technique, has a worse prognosis compared to their NPC counterparts, but with more significant esophagitis and mucositis. The disappointing long-term locoregional control intrigues interest on the detailed pattern of recurrence in these patients. Future studies focusing the location of locoregional relapse and radiation volume \& does may assist in determining the biological features of MPC and optimize treatments. Moreover, advanced radiation techniques may play a role in protecting normal tissues and reduce acute toxicities.

\section{Supplementary Material}

Supplementary table 1.

http://www.jcancer.org/v08p0563s1.pdf

\section{Acknowledgements}

This work was supported by Medical Scientific Research Foundation of Guangdong Province, China (No. B2014157).

\section{Competing Interests}

The authors have declared that no competing interest exists.

\section{References}

1. Kumagai $Y$, Kawano T, Nakajima $Y$, et al. Multiple primary cancers associated with esophageal carcinoma. Surg Today. 2001; 31: 872-6.

2. Lo OS, Law S, Wei WI, et al. Esophageal cancers with synchronous or antecedent head and neck cancers: a more formidable challenge? Ann Surg Oncol. 2008; 15: 1750-6.

3. Nagasawa S, Onda M, Sasajima K, et al. Multiple primary malignant neoplasms in patients with esophageal cancer. Dis Esophagus. 2000; 13: 226-30.

4. Poon RT, Law SY, Chu KM, et al. Multiple primary cancers in esophageal squamous cell carcinoma: incidence and implications. Ann Thorac Surg. 1998; 65: 1529-34.

5. Warren S, Gates O. Multiple primary malignant tumors. Am J Cancer. 1932; 16: $1358-414$.

6. Koide N, Yazawa K, Koike S, et al. Oesophageal cancer associated with other primary cancers: a study of 31 patients. J Gastroenterol Hepatol. 1997; 12: 690-4.

7. Utada M, Ohno $\mathrm{Y}$, Hori $\mathrm{M}$, et al. Incidence of multiple primary cancers and interval between first and second primary cancers. Cancer Sci. 2014; 105: 890-6.

8. Lee JS, Ahn JY, Choi KD, et al Synchronous second primary cancers in patients with squamous esophageal cancer: clinical features and survival outcome. Korean J Intern Med. 2016; 31: 253-9.

9. Lee GD, Kim YH, Kim JB, et al. Esophageal cancer associated with multiple primary cancers: surgical approaches and long-term survival. Ann Surg Oncol. 2013; 20: 4260-6.

10. Otowa Y, Nakamura T, Takiguchi G, et al. Safety and benefit of curative surgical resection for esophageal squamous cell cancer associated with multiple primary cancers. Eur J Surg Oncol. 2016; 42: 407-11.

11. Shinoto M, Shioyama $\mathrm{Y}$, Sasaki $\mathrm{T}$, et al. Clinical results of definitive chemoradiotherapy for patients with synchronous head and neck squamous cell carcinoma and esophageal cancer. Am J Clin Oncol. 2011; 34: 362-6.

12. Nguyen TD, Panis $X$, Legros $M$, et al. Neoadjuvant chemotherapy and irradiation in multiple synchronous squamous cell carcinoma of the upper aero digestive tract. Radiother Oncol. 1989; 16: 283-8.

13. Welz S, Schmid A, Hehr $\mathrm{T}$, et al. Treatment-outcome for synchronous head-and-neck and oesophageal squamous cell carcinoma. Radiother Oncol. 2005; 77: 267-70.

14. Wang WL, Wang YC, Chang CY, et al. Human papillomavirus infection on initiating synchronous esophageal neoplasia in patients with head and neck cancer. Laryngoscope. 2016; 126: 1097-102.

15. Wang WL, Lee CT, Lee YC, et al. Risk factors for developing synchronous esophageal neoplasia in patients with head and neck cancer. Head Neck. 2011; 33: 77-81.

16. Atienza JA, Dasanu CA. Incidence of second primary malignancies in patients with treated head and neck cancer: a comprehensive review of literature. Curr Med Res Opin. 2012; 28: 1899-909.

17. Jia $N$, Wen $X$, Zhang $N$, et al. Younger age of onset and multiple primary lesions associated with esophageal squamous cell carcinoma cases with a positive family history of the cancer suggests genetic predisposition. Chin Med J (Engl). 2014; 127: 2779-83.

18. Wen D, Wang S, Zhang L, et al. Early onset, multiple primary malignancies, and poor prognosis are indicative of an inherited predisposition to esophageal squamous cell carcinoma for the familial as opposed to the sporadic cases--an update on over 14-year survival. Eur J Med Genet. 2009; 52: 381-5.

19. Katada C, Muto M, Nakayama M, et al. Risk of superficial squamous cell carcinoma developing in the head and neck region in patients with esophageal squamous cell carcinoma. Laryngoscope. 2012; 122: 1291-6. 
20. Kuwabara T, Hiyama T, Tanaka S, et al. Genetic pathways of multiple esophageal squamous cell carcinomas. Oncol Rep. 2011; 25: 453-9.

21. Kuss I, Saito T, Johnson J, et al. Clinical significance of decreased $\zeta$ chain expression in peripheral blood lymphocytes of patients with head and neck cancer. Clin Cancer Res.1999; 5: 329-34.

22. Morita M, Kawano H, Otsu H, et al. Surgical resection for esophageal cancer synchronously or metachronously associated with head and neck cancer. Ann Surg Oncol. 2013; 20: 2434-9.

23. Wind $\mathrm{P}$, Roullet $\mathrm{MH}$, Douard $\mathrm{R}$, et al. Experience in the treatment of synchronous and metachronous carcinoma of the oesophagus and the head and neck. J Surg Oncol. 2000; 73: 138-42.

24. Wang WL, Chang WL, Yeh YC, et al. Concomitantly elevated serum matrix metalloproteinases 3 and 9 can predict survival of synchronous squamous cell carcinoma of the upper aero-digestive tract. Mol Carcinog. 2013; 52: 438-45.

25. Graff $P$, Schipman B, Desandes E, et al. Management of patients with head and neck tumours presenting at diagnosis with a synchronous second cancer at another anatomic site. Clin Oncol (R Coll Radiol). 2011; 23: 174-81. 